\title{
ANÁLISE DO REFINO DE GRÃO DE ALUMÍNIO PURO FUNDIDO EMPREGANDO Al-5Ti-1B, E SAIS DE KBF 4 E $\mathrm{K}_{2} \mathrm{TiF}_{6}{ }^{*}$
}

Marlon José Zanotto

Aleir Fontana De Paris²

\begin{abstract}
Resumo
O objetivo deste trabalho é comparar o uso da liga mãe Al-5Ti-1B e dos sais de fluortitanato de potássio $\left(\mathrm{K}_{2} \mathrm{TiF}_{6}\right)$ e fluorborato de potássio $\left(\mathrm{KBF}_{4}\right)$ no refino de uma liga de alumínio de pureza comercial. Os resultados demostram a eficiência da ligamãe no refino de grão quando adicionada em quantidades acima de $0,6 \% \mathrm{em}$ peso. No entanto, para o sal de boro, não houve refino de grão na mesma quantidade e no dobro da porcentagem. Já para o sal de titânio ocorreu refino de grão, provavelmente devido à presença de boro residual no alumínio puro comercial.

Palavras-chave: Refino de alumínio puro; Liga Al-5Ti-1B; Sais de $\mathrm{K}_{2} \mathrm{TiF}_{6} / \mathrm{KBF}_{4}$.

\section{ANALYSIS OF PURE CAST ALUMINUM GRAIN REFINING EMPLOYING Al-5Ti-1B, KBF 4 AND K2 $\mathrm{TiF}_{6} \mathrm{SALTS}^{*}$}

\section{Abstract}

To compare the influence on refining of commercial purity aluminum master alloy Al5Ti-1B and both fluortitanato (K2TiF6) and fluoroborate (KBF4) potassium salts are used in this study. The results demonstrated the grain refining efficiency when amounts above $0.6 \%$ by weight are added. For boron salt, no grain refinement it occurred in the same and double percentage. Grain refinement occurred with titanium salt, but probably due to the presence of residual boron in commercially pure aluminum.

Keywords: Pure aluminum refining; Al-5Ti-1B master alloy; $\mathrm{K}_{2} \mathrm{TiF}_{6} / \mathrm{KBF}_{4}$ salts.

1 Engenharia Mecânica, Acadêmico, DEM, Universidade Federal de Santa Maria, Santa Maria, RS Brasil,marlon_zanotto@hotmail.com

2 Engenharia Mecânica, Dr. Ing., Prof. Tit., DEM, Universidade Federal de Santa Maria, Santa Maria, RS Brasil,paris@ufsm.br 


\section{INTRODUÇÃO}

Na fundição de alumínio a modificação da estrutura dendrítica para uma estrutura de grãos finos do tipo equiaxial é desejada para melhorar as propriedades mecânicometalúrgicas da peça. Este tipo de estrutura apresenta várias vantagens tais como a minimização da contração, eliminação das trincas a quente, menor porosidade, melhor resistência à tenacidade, além de facilitar a laminação e/ou usinagem do fundido $[1,2]$.

Para se obter este refinamento da estrutura da peça tornou-se prática comum nas indústrias o uso de inoculação química [3]. O refinador de grão mais empregado para ligas de alumínio é a liga-mãe Al-5Ti-1B, que é composta de partículas do tipo $\mathrm{Al}_{3} \mathrm{Ti}$ e $\mathrm{TiB}_{2}$ em matriz de $\alpha$-Al. Este inoculante tem uma proporção Ti/B de $5 / 1$ devido a estudos realizados [4] que sugerem que o composto estequiométrico $\mathrm{TiB}_{2}$ (2,2\% em peso) seria o formador dos núcleos e necessitaria, para um bom refinamento, uma quantidade adicional de titânio, neste caso 2,8\% a mais.

Apesar de bastante empregado o uso dos inoculantes a base de Ti-B ainda deixa margens de dúvidas com discrepâncias nos resultados obtidos. Várias teorias foram propostas para explicar o funcionamento do inoculante na estrutura das peças fundidas em ligas de alumínio, porém sem um consenso sobre o mecanismo exato de refinamento usando Al-Ti-B [5]. Uma das mais antigas [6] propôs que as partículas de $\mathrm{TiB}_{2}$ são insolúveis no alumínio e funcionariam como sítios de nucleação heterogênea, a partir das quais se solidificaria $\alpha$-Al.

Para Mohanty e Gruzlesky [7] os boratos seriam deslocados para o contorno dos grãos e não funcionariam sem a presença do soluto titânio. Na teoria da nucleação peritética a mesma seria feita pelas partículas de $\mathrm{Al}_{3} \mathrm{Ti}$ que nucleariam $\alpha$-Al pela reação peritética [8]. Já Davies et al [9] publicaram que as partículas de $\mathrm{Al}_{3} \mathrm{Ti}$ seriam o centro do grão e desta forma o nucleante. No caso do refinamento do grão de ligas de alumínio com Al-Ti-B, a quantidade de Ti adicional no banho é normalmente menor que $0,01 \%$ o que seria muito baixa para a quantidade requerida para a reação peritética, $0,15 \%$ [4]. As partículas de $\mathrm{TiB}_{2}$ poderiam nuclear $\alpha$-Al, mas teriam uma potência moderada.

De acordo com [6] seria formada uma fina camada de $\mathrm{Al}_{3} \mathrm{Ti}$ na superfície das partículas de $\mathrm{TiB}_{2}$ que iniciaria a nucleação de $\alpha$-Al e na sequência seria dissolvida no alumínio através da reação peritética. As partículas de $\mathrm{TiB}_{2}$ somente seriam efetivas na nucleação de $\alpha$-Al com uma quantidade de titânio no banho fundido acima de 68 pppm.

Em uma revisão bibliográfica Quested [5] compara a influência separada da liga-mãe $\mathrm{Al}-\mathrm{Ti}$ e Al-B no refinamento de grão. A incorporação de quantidades pequenas de titânio $(0,01 \%)$ no alumínio fundido pode reduzir drasticamente o tamanho do grão. Uma reação peritética surge dentro do sistema alumínio-titânio:

$$
\text { Líquido }+\mathrm{Al}_{3} \mathrm{Ti} \rightarrow \alpha-\mathrm{Al} \text { (solução sólida) (1) }
$$

Como o $\mathrm{Al}_{3} \mathrm{Ti}$ tem uma estrutura tetragonal baseada em uma estrutura ordenada cúbica de face centrada, seria estável em fundidos contendo mais do que $0,15 \%$ de titânio. No caso de Al-B, apesar de pouco empregado, também ocorreria o refino. Este sistema apresentaria o eutético:

$$
\text { Líquido } \rightarrow \mathrm{AlB} 2+\alpha-\mathrm{Al} \text { (solução sólida) (2) }
$$

No caso da adição conjunta de Ti e B o refinamento de grão melhoraria de forma acentuada.

Segundo Sigworth e Kuhn [10], o refinador ativo seria na realidade o boro, pois permitira formar partícula de $\mathrm{TiB}_{2}$ que seria o responsável pela nucleação. 
Recentemente Wang et al $[11,12,13]$ estudaram o efeito no refinamento dos grãos de alumínio puro com adição da liga-mãe Al-3B, já que para alguns autores isto não ocorreria $[14,15]$ enquanto que para outros funcionaria somente em níveis elevados de adição $[16,17]$. Os resultados indicaram que somente seria efetiva a nucleação com partícula grandes de $\mathrm{AlB}_{2}$ e também em quantidade suficiente de impurezas para restringir o crescimento iniciais das dendrítas.

Neste trabalho se procurou fazer um estudo comparativo entre a liga-mãe comercial Al-5Ti-B e os sais $\mathrm{KBF}_{4}$ e $\mathrm{K}_{2} \mathrm{TiF}_{6}$ e sua influência no refino de grão de um lingote fundido em alumínio de pureza comercial.

\section{MATERIAIS E MÉTODOS}

O alumínio empregado nos experimentos foi de pureza comercial $(99,7 \%)$, com a composição química mostrada na Tabela 1, obtido por espectrometria de emissão ótica.

Tabela 1. Composição química do alumínio utilizado

\begin{tabular}{ccccccccc}
\hline Al \% & Si \% & Fe \% & Cu \% & Mg \% & Zn \% & Ti \% & B \% & Outros \\
\hline 99,73 & 0,082 & 0,145 & 0,033 & 0,0021 & 0,011 & $<0,002$ & 0,0097 & 0,015 \\
\hline
\end{tabular}

A quantidade de alumínio fundida foi 150 gramas, em função do molde utilizado, pesada em uma balança marca Kern, modelo 440-53, com capacidade máxima de $6000 \mathrm{~g}$ e precisão de \pm 1 grama. De acordo com a literatura consultada $[4,5]$ foram utilizadas as quantidades de inoculante de 0,$5 ; 0,75 ; 0,85 ; 1,00 ; 1,10 ; 1,20 ; 1,30$ e 1,50 gramas, correspondente a uma porcentagem de 0,3 a $1,0 \%$ do alumínio. A Tabela 2 ilustra a composição química fornecida pelo fabricante da liga-mãe.

Tabela 2. Composição química da liga Al-5Ti-1B

\begin{tabular}{ccccccc}
\hline Ti $\%$ & B $\%$ & V \% & Fe $\%$ & Si $\%$ & Outros total $\%$ & Al $\%$ \\
\hline 4,88 & 0,90 & 0,01 & 0,22 & 0,09 & 0,10 máx & balanço \\
\hline
\end{tabular}

No caso dos sais, a quantidade foi pesada em uma balança de precisão marca Kern, modelo 430-21, com capacidade máxima de 50 gramas e precisão de $\pm 0,001$ gramas.

Para efeito comparativo, na utilização dos sais de fluortitanato de potássio $\left(\mathrm{K}_{2} \mathrm{TiF}_{6}\right)$ e fluorborato de potássio $\left(\mathrm{KBF}_{4}\right)$, foram mantidas as proporções de $5 \%$ de Ti para $1 \%$ de Boro para verificar o comportamento dos sais de forma independente e também a mistura de ambos no alumínio fundido. Deste modo, partiu-se de um peso de 1 grama para facilidade de cálculo.

Para o sal $\mathrm{KBF}_{4}$ (fluorborato de potássio com pureza de 99,47\%), calculando a massa atômica dos elementos presentes $(K=39,10 ; B=10,81 ; F=19,00)$ encontrou-se o valor de 125,91 que em 1 grama do sal corresponde a 0,0864 gramas de boro. Para manter a mesma quantidade de boro da liga-mãe $0,01 \mathrm{~g} / 150 \mathrm{~g}$ de alumínio é necessário $0,12 \mathrm{~g}$ de sal. $\mathrm{O}$ mesmo cálculo foi aplicado ao sal $\mathrm{K}_{2} \mathrm{TiF}_{6}$ (fluortitanato de potássio com pureza de 97,71\%) $(\mathrm{Ti}=47,90)$. O peso de titânio em $1 \mathrm{~g}$ de sal é de 0,1995 e, com relação a liga-mãe $0,05 \mathrm{~g} / 150 \mathrm{~g}$ de alumínio é necessário $\sim 0,25 \mathrm{~g}$ de sal. Os cálculos foram efetuados sobre uma pureza considerada de $100 \%$.

A mistura dos dois sais também mantiveram a proporção de 5Ti/1B. 
O alumínio de pureza comercial foi fundido em um forno de aquecimento por resistência elétrica, na temperatura de $800^{\circ} \mathrm{C}$, em cadinho comercial de SiC. Após fusão e retirada da escória, o inoculante foi adicionado e o banho agitado durante um tempo de 30 segundos com uma vareta de grafite, sendo imediatamente retirado do forno e vazado em uma lingoteira de aço carbono com as dimensões em milímetro indicadas na Figura 1.

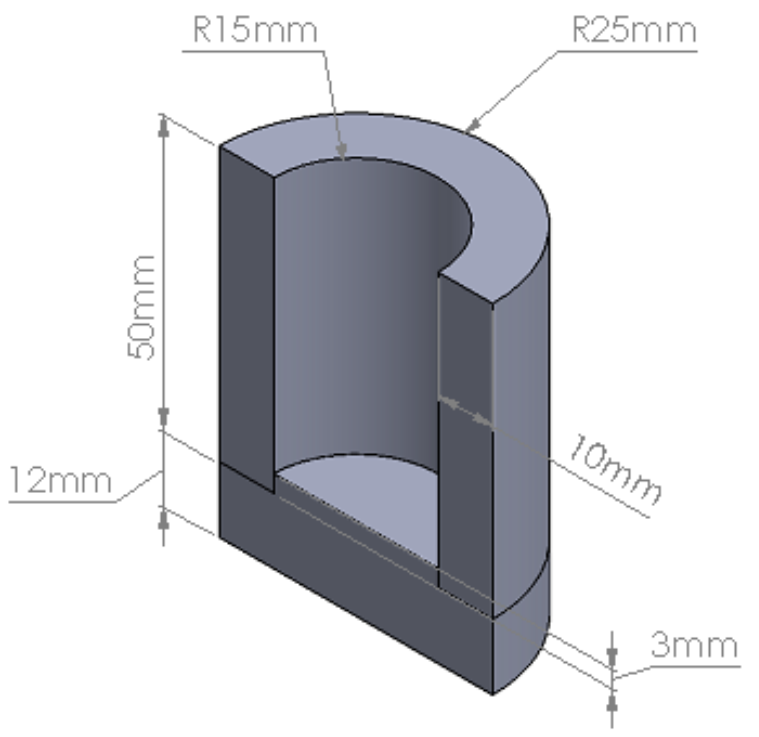

Figura 1. Dimensões da lingoteira.

A Tabela 3 mostra cada experimento com o tipo de refinador e o peso dos inoculantes utilizados. Para cada amostra foram fundidos dois lingotes para verificar a repetibilidade.

Tabela 3. Tipo de inoculante e peso utilizados nos experimentos

\begin{tabular}{cccc}
\hline Amostra & Refinador & Peso [gramas] & Relação Ti/B \\
\hline CP01 & - & - & - \\
\hline CP02 & Al-5Ti-1B & 0,75 & \\
\hline CP03 & Al-5Ti-1B & 0,85 & \\
\hline CP04 & Al-5Ti-1B & 1,0 & \\
\hline CP05 & Al-5Ti-1B & 1,1 \\
\hline CP06 & Al-5Ti-1B & 1,5 \\
\hline CP07 & KBF 4 & 0,12 & \multirow{2}{*}{$5 / 1$} \\
\hline CP08 & KBF $_{4}$ & 0,24 & \\
\hline CP09 & $\mathrm{K}_{2}$ TiF $_{6}$ & 0,25 & \\
\hline CP10 & $\mathrm{K}_{2}$ TiF $_{6}$ & 0,50 & \\
\hline CP11 & $\mathrm{KBF}_{4}+\mathrm{K}_{2} \mathrm{KiF}_{6}$ & $0,12+0,25$ \\
\hline CP12 & $\mathrm{KBF}_{4}+\mathrm{K}_{2} \mathrm{KiF}_{6}$ & $0,24+0,50$ \\
\hline
\end{tabular}

As peças fundidas foram analisadas por espectrometria de emissão ótica. Para a espectrometria foram efetuadas 4 "queimas" para verificar a repetibilidade. Os resultados que serão apresentados são a média dos valores. 


\section{RESULTADOS E DISCUSSÃO}

Após resfriamento as amostras foram cortadas aproximadamente na metade do lingote, lixadas, polidas e atacadas com reativo de Tucker para revelar a macroestrutura de solidificação. A Figura 2 (a-f) mostra as imagens obtidas do alumínio puro (a) e com as adições crescentes da liga-mãe nas proporções de 0,75 até 1,5 gramas. Com relação as estruturas obtidas, a partir da adição de $1,5 \mathrm{~g}$ do refinador, o comportamento mostrou-se inalterado nas condições empregadas.

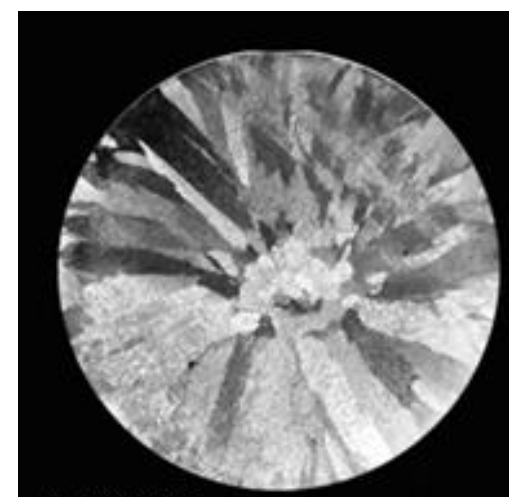

(a) Al puro (b) $0,75 \mathrm{~g}$

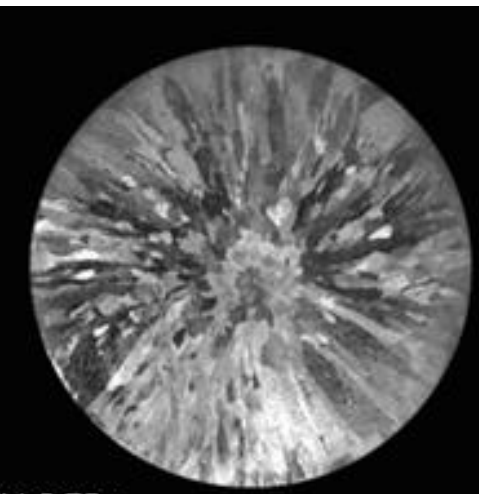

(b) 0,759

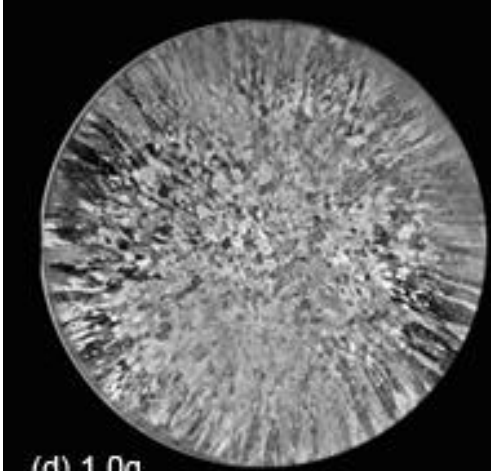

(d) $1,0 \mathrm{~g}$

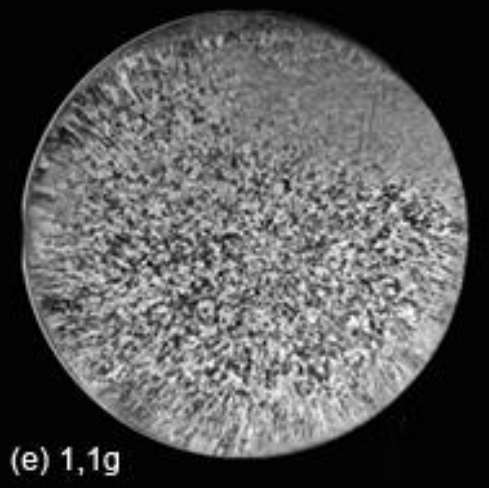

(e) $1,1 \mathrm{~g}$

\section{(c) $0,85 \mathrm{~g}$}
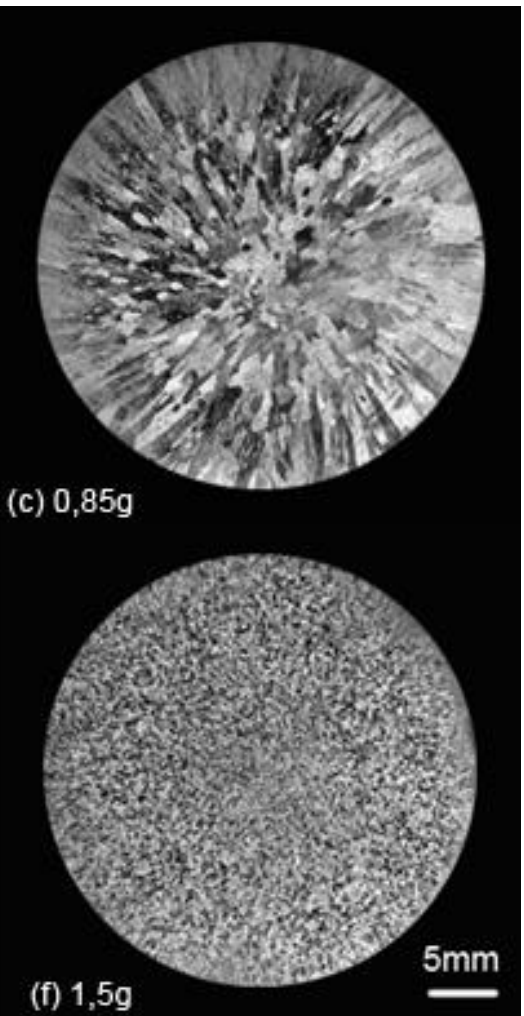

Figura 2. Macrografia da seção transversal dos lingotes de Al puro e com adições de Al-5Ti-1B.

O refino aumentou com o aumento da quantidade de refinador. Este resultado está conforme a literatura [5] e comprava o que já era esperado devido a eficácia da ligamãe Al-5Ti-1B.

A composição química das amostras, obtidas por espectrometria de emissão ótica, com 1,0 e 1,5g está mostrada na Tabela 4

Tabela 4. Composição química com adição da liga-mãe com 1,0 e 1,5g, CP04 e 06

\begin{tabular}{ccccc}
\hline Amostra & Al\% & Ti\% & B\% & Outros \\
\hline CP04 & 99,69 & 0,028 & 0,013 & 0,269 \\
\hline CP06 & 99,68 & 0,047 & 0,017 & 0,256 \\
\hline
\end{tabular}

Com estes resultados, chega-se a relação de Ti/B igual a 2,15, para amostra CP04. Da mesa forma, para a amostra CP06, relação Ti/B é de 2,76. Isto implicaria no refino de grão, como comprovado pelas Figuras $2 \mathrm{~d}$ e $2 \mathrm{f}$, mesmo sem uma quantidade apreciável de excesso de titânio acima da composição eutética do $\mathrm{TiB}_{2}$ de $2,2 \%$ de titânio.

$\mathrm{Na}$ utilização de sal de fluorborato de potássio $\left(\mathrm{KBF}_{4}\right)$, a adição de $0,12 \mathrm{~g}$, Figura 3a, não mostrou nenhuma alteração notável com relação a macroestrutura do alumínio 
puro, Figura 2a. Em ambas as imagens, verifica-se uma estrutura colunar dendrítica formada a partir da parede da lingoteira devido à extração do calor, e alguns grãos equiaxiais no centro do lingote como consequência do equilíbrio térmico das frentes de solidificação.

Considerando que talvez a pequena quantidade de sal $(0,12 \mathrm{~g})$ pudesse influenciar a solidificação, esta quantidade foi dobrada $(0,24 \mathrm{~g})$ para o mesmo volume de alumínio fundido, Figura 3b. Conforme pode ser analisado pelas imagens, esta variação não mostrou nenhuma influência significativa na cristalização/refinamento do lingote de alumínio. Talvez uma pequena alteração na espessura das dendrítas.

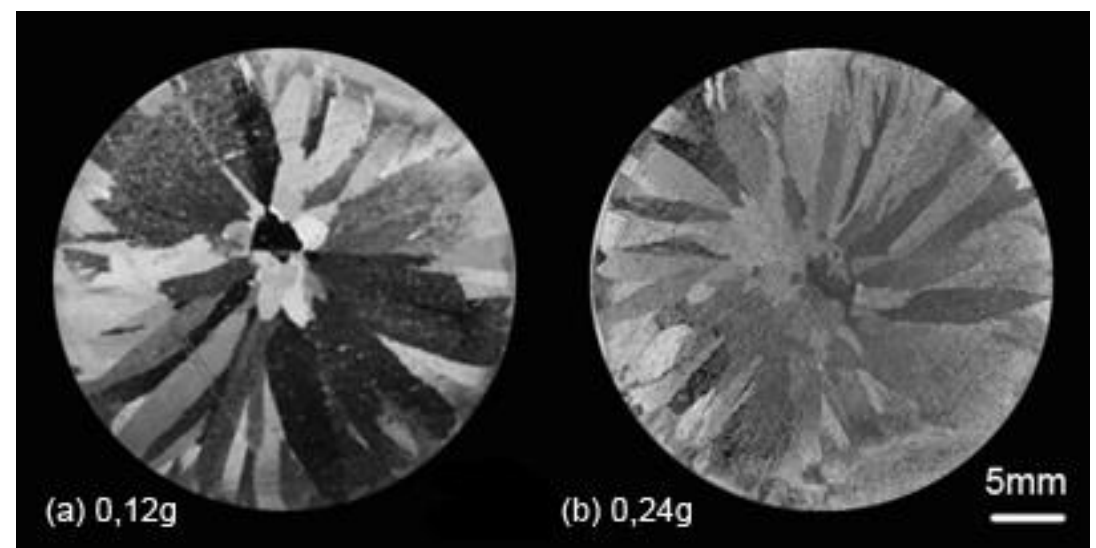

Figura 3. Macroestrutura de solidificação do alumínio com adições de 0,12g(a) e 0,24(b) do sal KBF 4 .

Os resultados da espectrometria das amostras com adições de sal de fluorborato de potássio $\left(\mathrm{KBF}_{4}\right)$, estão mostradas na Tabela 5.

Tabela 5. Composição química com adição de sal $\mathrm{KBF}_{4} 1 \mathrm{~g}$ e $2 \mathrm{~g}$

\begin{tabular}{ccccc}
\hline Amostra & Al\% & Ti\% & B\% & Outros \\
\hline CP07 & 99,74 & $<0,002$ & 0,015 & 0,243 \\
\hline CP08 & 99,76 & $<0,002$ & 0,016 & 0,222 \\
\hline
\end{tabular}

Efetuando o cálculo chega-se aos seguintes resultados para a relação Ti/B: 0,13 e 0,12 . Os resultados da Figura $3 a$ e $3 b$ mostram que não houve refino de grãos o que confere com a literatura [7]. Assim, pode-se constatar que o boro não teria potência nucleadora quando aplicado no alumínio de pureza comercial utilizado neste trabalho, talvez pela não formação de $\mathrm{TiB}_{2}$ devido a falta de titânio para completar a estequiometria.

Empregando a mesma metodologia foram fundidos dois lingotes com adição de $0,25 \mathrm{~g}$ de sal de fluortitanato de potássio $\left(\mathrm{K}_{2} \mathrm{TiF}_{6}\right)$, Figura $4 \mathrm{a}$, e $0,50 \mathrm{~g}$, Figura 4b. Como pode ser observada a macroestrutura apresentou um refino acentuado para os dois casos. Na Figura $4 \mathrm{a}$, correspondente a mesma quantidade de $\mathrm{Ti} / \mathrm{B}$ empregada no refino com a liga-mãe Al-5Ti-1B (Figura 2d). Com o aumento do teor de titânio o refino foi mais acentuado, Figura 4b. Neste caso resta uma dúvida se 0 titânio seria refinador de grão independente do boro ou, como existe boro residual $(\sim 0,010 \%)$ este boro ajudaria na formação de $\mathrm{TiB}_{2}$, já que a relação entre $\mathrm{Ti} / \mathrm{B}$ é maior do que a quantidade estequiométrica. 


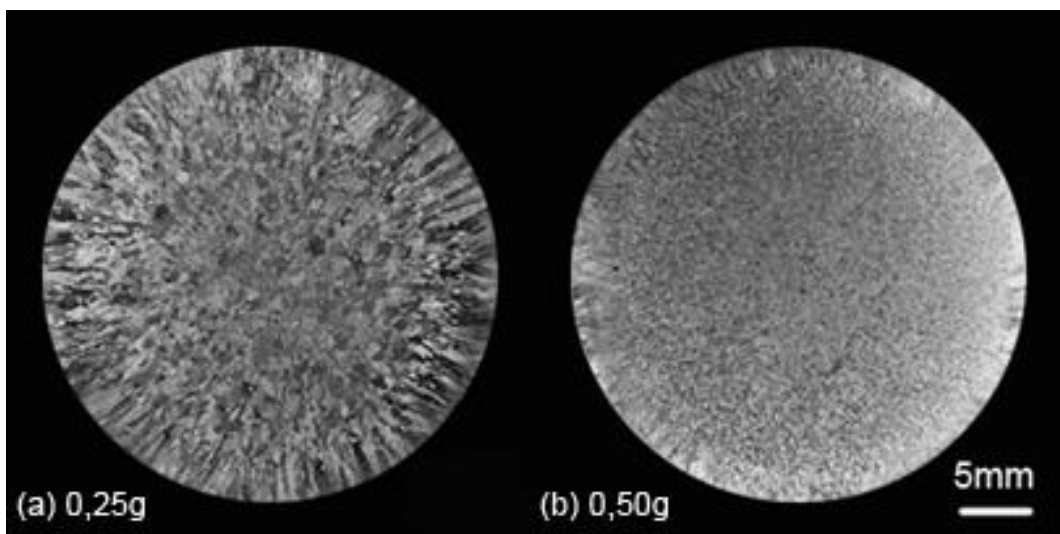

Figura 4. Macroestrutura de solidificação com alumínio com adições de $0,24 \mathrm{~g}(\mathrm{a})$ e $0,50 \mathrm{~g}(\mathrm{~b})$ do sal $\mathrm{K}_{2} \mathrm{TiF}_{6}$.

Os resultados da espectrometria estão mostrados na Tabela 6.

Tabela 6. Composição química com amostras de sal $\mathrm{K}_{2} \mathrm{TiF}_{6} 1 \mathrm{~g}$ e $2 \mathrm{~g}$

\begin{tabular}{ccccc}
\hline Amostra & Al\% & Ti\% & B\% & Outros \\
\hline CP09 & 99,72 & 0,028 & 0,0088 & 0,243 \\
\hline CP10 & 99,69 & 0,055 & 0,0088 & 0,246 \\
\hline
\end{tabular}

Nestas amostras o teor de boro ficou abaixo daquele encontrado para o alumínio puro. Conforme El-Mahallawy et al [17] isto pode acontecer devido a limitações da cinética e transporte de massa no sistema. Fazendo a relação direta, ou seja, considerando os valores do boro da tabela, a relação Ti/B seria de 3,18 e 6,25. Esta situação seria explicada em ambos os casos pela quantidade necessária para formar o partículas estequiométricas de $\mathrm{TiB}_{2}$.

O resultado da adição da mistura dos dois sais, mantendo a mesma proporção da liga-mãe Al-5Ti-1B, está mostrado na Figura 5. Seguiu-se a mesma metodologia de adição e tempo de agitação do banho de $30 \mathrm{~s}$, conforme os experimentos precedentes. No primeiro caso, Figura $5 \mathrm{a}$, foi adicionado $0,12 \mathrm{~g}$ de fluorborato de potássio $\left(\mathrm{KBF}_{4}\right)$ e $0,25 \mathrm{~g}$ de fluortitanato de potássio $\left(\mathrm{K}_{2} \mathrm{TiF}_{6}\right)$, e no segundo caso, Figura $5 \mathrm{~b}$, o dobro da quantidade $0,24 \mathrm{~g}+0,50 \mathrm{~g}$, sempre na proporção de $5 \mathrm{Ti} / 1 \mathrm{~B}$.

Analisando as imagens, pode-se verificar que com a quantidade empregada da mistura de sais, correspondente a $1 \mathrm{~g}$ da liga-mãe Al-5Ti-B, não ocorreu refinamento de grão, enquanto que para o aumento para $2 \mathrm{~g}$ houve refinamento se assemelhado a imagem da Figura $2 d$.

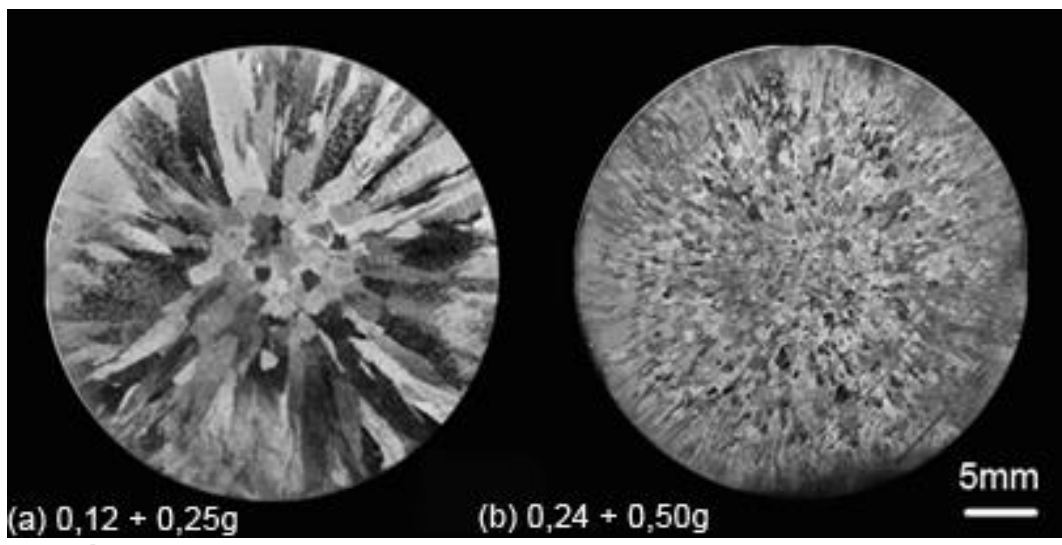

Figura 5. Macroestrutura com adição dos sais $\mathrm{KBF}_{4} \mathrm{e} \mathrm{K}_{2} \mathrm{TiF}_{6}$. 
Para a mistura dos sais $\left(\mathrm{KBF}_{4}\right.$ e $\left.\mathrm{K}_{2} \mathrm{TiF}_{6}\right)$ a Tabela 7 fornece os valores da composição química.

Tabela 7. Composição química com as misturas de sais

\begin{tabular}{ccccc}
\hline Amostra & Al\% & Ti\% & B\% & Outros \\
\hline CP11 & 99,71 & 0,029 & 0,014 & 0,247 \\
\hline CP12 & 99,70 & 0,037 & 0,014 & 0,249 \\
\hline
\end{tabular}

Efetuando o cálculo da relação Ti/B na liga, chega-se aos seguintes valores para a relação Ti/B: 2,07 e 2,64. Analisando as imagens da Figura 5a e 5b, verifica-se que ocorreu o refino de grão do lingote fundido somente com a quantidade maior de sal. No primeiro a quantidade de titânio não teve um excesso para formar uma quantidade suficiente de partículas estequiométricas $\mathrm{TiB}_{2}$. No segundo caso foi possível atingir a porcentagem estequiométrica.

\section{CONCLUSÃo}

Dos resultados acima apresentados e discutidos, se chegam a algumas conclusões sobre o trabalho experimental. O refino de grão com liga mãe Al-5Ti-B mostrou ser um eficiente refinador em quantidades acima de 0,6\%, Valores superiores não mostraram modificações sensíveis. O boro, sem a presença de titânio, não funciona como refinador de grão para alumínio com pureza acima de 99,7\%. A presença de uma quantidade de titânio acima da porcentagem estequiométrica $\mathrm{TiB}_{2}$ é necessária para formar núcleos insolúveis para início da solidificação. O titânio, sem a presença de boro, apresentou um bom refino de grão, mas na pureza do alumínio comercial, empregado com a presença de boro residual, apresenta uma quantidade acima da composição estequiométrica o que poderia ter como consequência este resultado.

\section{Agradecimentos}

Os autores gostariam de prestar seu agradecimento a LSM do Brasil pelo fornecimento da liga-mãe e dos sais utilizados nos experimentos. Também o sincero agradecimento ao Dr. Eng. Márcio Kramer de Macedo do LAMEF-UFRGS pela espectrometria de emissão ótica.

\section{REFERÊNCIAS}

1 Metals Handbook. Casting. 3를 Edição, Materials Park, Ohio, ASM, 1996, p. 750.

2 Easton M, StJohn D. Grain refinement os aluminum alloys: Part I. The solute nucleante and solute paradigms - A review of the literature. Metal. Material Trans. A. 1999(30A):1613-1624.

3 Murty BS, Sakori AS, Chakaraborty M. Grain refinement of aluminium ant its alloys by heterogeneous nucleation alloying. Int. Mat. Rev. 2002; 47(1): 3-29.

4 Fan Z, et al. Grain refinement mechanism in the Al/AI-Ti-B. Acta Materialia, 2015(84): 292-304.

5 Quested TE. Understanding mechanisms of grain refinement of aluminium alloys by inoculation. Mat. Sci. Tech. 2004;20(11): 1357-1369.

6 Cibula A. J. Inst. Metals. 1949-50(76):321-360, APUD [5].

7 Mohanty PS, Gruzleski JE. Mechanism of grain refinement in aluminium. Acta Metall. Mat. 1995; 43(5): 2001-2012.

8 Crosley PE; Mondolfo LF. The modification of aluminum-silicon alloys. AFS Trans. 1966(74): 53-64. 
9 Davies IG, Dennis JM, Hellawell A. Nucleation of aluminum grain in alloys of aluminum with titanum and boron. Metall. Mater. Trans. 1970;1(1): 275-280.

10 Sigworth GK; Kuhn TA. Grain refinement of aluminum casting alloys. AFS Tras. Paper a7-067(02), 2007: 1-12

11 Wang X, Liu Z, Dai W. Han Q. On the mechanism of aluminum grain refinement by AlTi-B type master alloy. Metall. Mater. Trans. B. 2015; 46B(8): 1620-1625.

12 Wang T. et al. Grain refinement of pure aluminum by inoculation with Al-B master alloys. Mater. Sci. Eng. A. 2012(549):136-143.

13 Wang T et al. Grain refinement potency of Al-B máster alloy on pure aluminum. Scipta Materialia. 2011(64): 1121-1124.

14 Sigworth GK, Guzowski MM. Grain refining of hypo-eutectic Al-Si alloys," AFS Trans. 1985(93): 907-912.

15 Kori SA, Murty BS, Chakraborty M. Development of an efficient grain refiner for Al - 7Si alloy and its modification with strontium. Mater. Sci. Eng. A. 2000(A283): 94-104.

16 Vinod Kumar GS, Murty BS, Chakraborty, M. Effect of $\mathrm{TiAl}_{3}$ particles size and distribution on their settling and dissolution behaviour in aluminium. J. Mater. Sci. 2010(45):2921-2929.

17 El-Mahallawy $\mathrm{N}$ et al. On the reaction between aluminium, $\mathrm{K}_{2} \mathrm{TiF}_{6}$ and $\mathrm{KBF}_{4}$. J. of Alloys and Compounds 1999(292):221-229. 